\title{
The great imitator
}

\author{
Cristina Gozzi, Maurizio Tognetti, Lucio Brugioni \\ Medicina Interna Area Critica, AOU Policlinico di Modena, Italy
}

\begin{abstract}
B.K. a Moroccan 32-year-old man was admitted to our hospital for abdominal pain and vomiting. He showed an increase of inflammation indexes, abdominal free fluid, axillary, inguinal and abdominal lymphadenitis, pleural effusion, ascites and thickened intestinal walls. The patient also presented alopecia and facial erythema, a positive anti-nuclear antibodies (ANA) test (1:1280). Lastly the diagnosis of systemic lupus erythematosus (SLE) was made. The patient was treated with high doses of corticosteroid; abdominal pain decreased and pleural effusion and ascites disappeared. After several days he started to complain of significant chest pain. He had a troponin increased above $100 \mathrm{ng} / \mathrm{mL}$, modified electrocardiogram, pericardial effusion and edema of ventricular walls as in myocarditis. The patient was treated successfully with cyclophosphamide and pericardial effusion and chest pain disappeared. After 1 month at the hospital the patient was discharged and referred to a rheumatologist for the follow up. Systemic lupus erythematosus is an autoimmune disease in which autoantibodies and immune-complexes damage heart, joints, skin, blood vessels, liver, kidneys and the nervous system. Ninety percent of individual diagnosed with SLE are women. The course is unpredictable with periods of acute illness alternating with periods of remission. This is a typical disease that requires a differential diagnosis, because the symptoms may vary widely and are unpredictable. According to the American College of Rheumatology, which drew up a reference list of 11 symptoms, SLE is confirmed when 4 out of the 11 symptoms are present simultaneously or symptoms overlap on two separate occasions. Treatment can include corticosteroids and antimalarial drugs, intravenous immunoglobulins and cytotoxic drugs such as cyclophosphamide. Our case is exceptional because it is rare for a young man to be affected. He had 5 out of 11 symptoms: malar rash, serositis, arthritis, hematologic disorder and ANA test positive.
\end{abstract}

\section{Case Report}

B.K., a 32-year-old Moroccan man, was admitted to our department of internal medicine for abdominal pain and vomiting. He reported loss of weight $(15 \mathrm{~kg}$ over 8 months) and migrant arthritis of the joints.

On admission his vital signs were normal, while on examination a decreased vesicular murmur was heard bilaterally in the lower part of the lungs. Diffused tenderness of the abdomen, alopecia and facial erythema were also found.

Correspondence: Gozzi Cristina, Medicina Interna Area Critica, AOU Policlinico di Modena, via del Pozzo 71, Modena, Italy. Tel.: +39.059.4225491.

E-mail: gozzic@libero.it

Key words: systemic lupus erythematosus, autoimmune disease.

Received for publication: 12 September 2013.

Revision received: 23 December 2013.

Accepted for publication: 20 January 2014

This work is licensed under a Creative Commons Attribution NonCommercial 3.0 License (CC BY-NC 3.0).

(C) Copyright C. Gozzi et al., 2014

Licensee PAGEPress, Italy

Italian Journal of Medicine 2014; 8:189-192

doi:10.4081/itjm.2014.430
Blood exams showed a normocytic and normochromic anemia (hemoglobin $10.5 \mathrm{~g} / \mathrm{dL}$ and mean corpuscular volume $80 \mathrm{fL}$ ) and an increase of inflammatory indexes. An abdominal ultrasound revealed copious free fluid. An x-ray of the abdomen showed some air fluid levels. A computed tomography scan of the thorax and of the abdomen confirmed ascites, pleural effusion, inguinal, axillary and abdominal lymphadenitis, thickness of some portions of the intestine. The patient was put on parenteral nutrition and an empiric antibiotic treatment was started.

He underwent an explorative laparoscopy that revealed no abnormal findings (no free fluid or thickness of the small intestine). The biopsy, done on some of the mesenteric lymph nodes taken during the laparoscopy procedure, revealed a slight inflammation of this tissue.

The blood exams excluded a potential microbiological etiology, such as HIV, QuantiFERON ${ }^{\circledR}$-TB (Cellestis Ltd., Carnegie, Victoria, Australia), Epstein-Barr virus, cytomegalovirus, Brucella, Leishmania. On the other hand anti-nuclear antibodies (ANA) (1:1280), extractable nuclear antibodies (ENA), DNA, anti-Smith (Sm) antibodies were positive; the complement system was modified (C3: 50 $\mathrm{mg} / \mathrm{dL}$; $\mathrm{C} 49 \mathrm{mg} / \mathrm{dL}$ ) and a hypothyroidism with an increased level of thyroglobulin antibodies was found $(3785 \mathrm{UI} / \mathrm{mL})$.

A biopsy of the facial skin (affected by rush) was also performed and led to a diagnosis of lupus erythematosus. 
Soon after the diagnosis, the patient was put on methylprednisolone [500 mg/die intravenously (i.v.) for three days and then $1 \mathrm{mg} / \mathrm{kg}$ ] and intravenous immunoglobulins (IVIGs), achieving a gradual remission of abdominal pain. A couple of days later, the patient complained of chest pain: there was an increase of troponin $(100 \mathrm{ng} / \mathrm{mL})$ in his blood level and the various electrocardiograms done in sequence showed Q waves.

The transthoracic echocardiogram showed a thickness of the left ventricle with no obstruction of the blood flow, hypokinesis of the septum and of the apex, an ejection fraction of $45 \%$, a small posterior pericardial effusion with no constrictive effect.

The cardiac magnetic resonance imaging confirmed the diagnosis of pericarditis and myocarditis with an irrelevant hypokinesis of the left ventricle (in anterior and septal segments) and a much more remarkable hypokinesis of the apex (anterior and inferior segment); in these segments in $\mathrm{T} 2$ sequences a higher intensity in tissue was noticed, as if to indicate myocardial edema. The pericardial effusion with a thickness of $1 \mathrm{~cm}$ was confirmed as well.

Therefore, in agreement with the rheumatologist, the patient was also treated with cytotoxic drugs: initially we started with $200 \mathrm{mg}$ of i.v. cyclophosphamide every other day and then we switched to $100 \mathrm{mg}$ orally.

Only then did we witness a gradual recovery of the patient: he no longer had inflammation of the serous membranes, on the echocardiogram follow up he had a normal global kynesis, he no longer complained of pain. He was then discharged and sent to the rheumatologists for the follow up.

\section{Discussion}

Systemic lupus erythematosus (SLE) is a systemic autoimmune disease (or autoimmune connective tissue disease) that can affect any part of the body. As can be seen in other autoimmune diseases, the immune system attacks the body cells and tissues, triggering inflammation and tissue damage. The disease occurs 9 times more often in women than in men. Statistics show that the prevalence of SLE in the United States ranges from 15 to 50 over 100,000 people. It manifests itself in young adults in their 20 s to 40 s, even though it can occur at any age. ${ }^{1}$

There is no one specific cause of SLE. There are, however, environmental triggers and genetic susceptibilities.

The onset can affect only one organ or be systemic involving several parts of the body.

SLE is one of several diseases that in the AngloSaxon countries are known as great imitators, because it often mimics or is mistaken for other illnesses. SLE is a classical item in differential diag- nosis, because SLE symptoms vary widely and come and go unpredictably.

The disease varies from imperceptible and intermittent signs and symptoms to a persistent condition that can bring to death rapidly. The course of the disease is unpredictable, with periods of illness (called flares) alternating with remissions. A complete recovery occurs in less than $20 \%$ of the patients. ${ }^{2,3}$

Common initial and chronic complaints include fever, joint pains, myalgia, fatigue, anorexia.

In general this disease involves more frequently the musculoskeletal system, kidneys, blood, skin and nervous system. ${ }^{4}$

The most common symptom is joint pain, in particular in the small joints of the hand (metacarpal-phalangeal joint $76 \%$, proximal inter-phalangeal joint $82 \%$ ) followed by wrists and knees. Muscle pain can occur in $50 \%$ of the patients. ${ }^{5}$

Half of the patients present dermatological signs: the classic malar rash (or butterfly rash) is an erythematosus rash that involves cheeks, nose, ears and chin. Some may have alopecia as well. Twenty percent of patients have Raynaud's phenomenon, which is an excessive vasomotor response to cold or more precisely a hyper-activation of the sympathetic nervous system causing extreme vasoconstriction of the peripheral blood vessels with blood stasis that has a spontaneous resolution.

The renal system can be injured in many ways and develop a range of disorders from a slight proteinuria, to painless hematuria, nephritic syndrome or renal failure. In most patients we can find a deposition of immune complex along the glomerular basal membrane; only about half of the patients develop glomerulonephritis and persistent proteinuria. ${ }^{6}$ Patients with diffused proliferative glomerulonephritis generally need a more aggressive therapy with cytotoxic drugs, because, if not treated rapidly and appropriately, they can develop renal failure. In these patients a renal biopsy can be helpful.

The nervous system can be damaged as well; in general when the disease has already involved other systems. We can have central nervous system disorders and peripheral nervous system disorders. The neurological symptoms include headaches, depression, seizures, cognitive dysfunction, mood and anxiety disorders, polyneuropaties, psychosis..$^{7-9}$

An SLE patient may have inflammation of various parts of the heart, such as pericarditis (generally the most common one), myocarditis, and endocarditis. Endocarditis in SLE is typically non-infective due to the sediment of immunocomplexes (Libman Sacks endocarditis), and involves either the mitral valve or the tricuspid valve. Pleural effusion can also be seen. ${ }^{10,11}$

Gastrointestinal involvement manifests itself with nausea, diarrhea, and abdominal pain. Vasculitis of the 
gastrointestinal tract is one of the most fearful complications.

The blood tests generally reveal normocytic and normochromic anemia, leukopenia and lymphopenia. In $25 \%$ of the cases we can find high levels in the venereal disease research laboratory test, due to the presence of anti-phospholipid antibodies (aPL). If this occurs, we generally define it as a false positivity for venereal diseases (syphilis).

Other tests routinely performed in suspected SLE patients aim to measure complement system levels, since there may be low levels of $\mathrm{C} 3$ and $\mathrm{C} 4$. The level of erythrocyte sedimentation rate is generally high.

ANA and anti ENA are the most important serologic tests for SLE. ANA screening may give positive results in many connective tissue disorders and other autoimmune diseases, and may occur in normal individuals as well. Subtypes of the ANA that are linked to SLE and are positive in $70 \%$ of the patients are the anti-double stranded DNA antibodies (dsDNA); subtypes of ENA that are linked to SLE include anti-Sm antibodies.

The prevalence of aPL ranges from $1 \%$ to $10 \%$ in the general population, $16 \%$ in patients with rheumatoid arthritis and 30 to $40 \%$ in patients with SLE.

The strength of the correlation between aPL and thrombosis varies among studies depending on type of aPL test, study design and clinical population studied.

Although any vasculature can be affected by thrombosis, stroke and transient ischemic attack are the most common presentations of arterial thrombosis, whereas deep vein thrombosis is the most common presentation of venous thrombosis.

The major cardiac manifestations of aPL include valve disease, myocardial infarction, intracardiac thrombosis. The commonest cardiac manifestation of aPL is valve abnormalities in the form of vegetations and thickening similar to Libman-Sacks endocarditis in SLE. Left-sided valves are involved more frequently; mitral valves are more commonly affected than aortic valves. The predominant functional abnormality is regurgitation, whereas stenosis is rare. Valve abnormalities are usually asymptomatic. Patients with valve abnormalities have a higher incidence of arterial embolism than those without valve disease. There is mounting evidence that aPL is associated with an increased risk of myocardial infarction. ${ }^{12}$

In 1982 the American College of Rheumatology (ACR) drew up a reference list of 11 criteria, revised in 1997, which is intended to be a classification tool to simplify the diagnosis that quite often is very complex. When 4 out of 11 criteria are present simultaneously or overlap on 2 separate occasions, the SLE diagnosis is confirmed.

The symptoms are: i) malar rash (rash on cheeks); ii) discoid rash (red, scaly patches on skin that cause scarring); iii) serositis: pleuritis, pericarditis; iv) oral ulcers (oral or nasopharyngeal ulcers); v) arthritis: non-erosive arthritis of two or more peripheral joints, with tenderness, swelling, or effusion; vi) photosensitivity (exposure to ultraviolet light causes rash, or other symptoms of SLE flare-ups); vii) hematologic disorders: hemolytic anemia (low red blood cell count) or leukopenia (white blood cell count $<4000 / \mu \mathrm{L})$, lymphopenia $(<1500 / \mu \mathrm{L})$ or thrombocytopenia; viii) renal disorders: more than $0.5 \mathrm{~g}$ per day of protein in urine or cellular casts seen in urine samples under a microscope; ix) positive ANA test; $x$ ) immunologic disorders: positive anti-Sm, antidsDNA, anti-phospholipid antibody, and/or false positive serological test for syphilis; presence of antisingle-stranded DNA in $70 \%$ of cases; xi) neurologic disorder: seizures or psychosis.

The treatment of SLE involves preventing flares and reducing their severity and duration when they occur. Low levels of $\mathrm{C} 3$ and high levels of antidsDNA antibodies are a negative prognostic factor and require an aggressive treatment.

Due to the variety of symptoms and organ systems involved in this disease, an individualized assessment of its severity is required to treat each patient successfully. A mild form of SLE may be treated with non-steroidal anti-inflammatory drugs (NSAIDs), sometimes associated with hydroxychloroquine which has been introduced in the treatment of SLE since 1955 for cutaneous and articular manifestations. Immunosuppressants, as methotrexate and cyclosporine, can be used as well: the former for a better control of joint involvement, and the latter for hematologic abnormalities. Severe cases with severe organ and hematologic complications can be treated with i.v. NSAIDs and cyclophosphamide, which are used in particular for severe glomerulonephritis. IVIGs may be helpful to control SLE with organ involvement, or vasculitis. ${ }^{13,14}$

A new treatment specifically targeted against SLE is belimumab. It is an IgG1 monoclonal antibody that inhibits the activity of the soluble cytokine B-lymphocyte stimulator, also known as B-cell activating factor. It is targeted to the fundamental pathology of SLE and has been widely regarded as a step change in treatment options. It was recently licensed in the USA and in Europe for the treatment of autoantibody-positive SLE. The European indication is for severely affected SLE patients with active, autoantibody-positive disease and a high degree of disease activity exemplified by positive anti-dsDNA and low complement despite standard therapy. Belimumab is administered by intravenous infusion recommended at $10 \mathrm{mg} / \mathrm{kg}$ on days 0,14 and 28 and subsequently at 28 -day intervals. ${ }^{15}$ 


\section{Conclusions}

The peculiarity of this case of SLE is that the patient was a young man and that the onset was abdominal pain with bowel obstruction. The laparoscopic procedure was not useful for the diagnosis of SLE, but was helpful in ruling out other causes of abdominal peritonitis. As the Anglo-Saxons say, SLE is the great imitator. Ascites, abdominal lymphadenitis and the thickness of some portions of the bowels had to be considered in the differential diagnosis with inflammatory bowel disease (Crohn's disease), systemic inflammation that can cause sieritis, like tuberculosis or peritoneal carcinosis. Moreover, when the patient started to have heart problems, a differential diagnosis was mandatory to understand if he had pericarditis, myocarditis or acute myocardial ischemia. Also, based on the SLE disease criteria, many of the patient's organs were damaged. In fact, he had 5 of the criteria listed by ACR (malar rash, serositis, non-erosive arthritis, hematologic disorder, positive anti-Sm, antidsDNA).

SLE is a great challenge for physicians, because this disease can involve many organs and systems. Only a thorough knowledge and a correct differential diagnosis can lead to the right diagnosis and an adequate medical therapy and patient management.

\section{References}

1. Rahman A, Isenberg DA. systemic lupus erythematosus. N Engl J Med 2008;358:929-39.

2. D'Cruz DP, Khamashta MA, Hughes GR. Systemic lupus erythematosus. Lancet 2007;369:587-96.
3. Mok CC, Lau CS. Pathogenesis of systemic lupus erythematosus. J Clin Pathol 2012;65:481-90.

4. Jump RL, Robinson ME, Armstrong AE, et al. Fatigue in systemic lupus erythematosus contributions of disease activity, pain, depression and perceived social support. J Rheumatol 2005;32:1699-705.

5. Mendoza-Pinto C, García-Carrasco M, Sandoval-Cruz $\mathrm{H}$, et al. Risk factors of vertebral fractures in women with systemic lupus erythematosus. Clin Rheumatol 2009;28:579-85.

6. Syuto T, Shimizu A, Takeuchi Y, et al. Association of antiphosphatidylserine/prothrombin antibodies with neuropsychiatric systemic lupus erythematosus. Clin Rheumatol 2009;28:841-5.

7. West SG. Lupus and the central nervous system. Curr Opin Rheumatol 1996;8:408-14.

8. Neuwelt CM, Young RG. Managing neuropsychiatric lupus: top 10 clinical pearls. J Muscoskel Med 2009;26:127-35.

9. Honczarenko K, Budzianowska A, Ostanek L. Neurological syndromes in systemic lupus erythematosus and the association with antiphospholipid syndrome. Neurol Neurochir Pol 2008;42:513-7.

10. Asanuma Y, Oeser A, Shintani AK, et al. Premature coronary artery atherosclerosis in systemic lupus erythematosus. N Engl J Med 2003;349:2407-14.

11. Hahn BH. Systemic lupus erythematosus and accelerated atherosclerosis. N Engl J Med 2003;349:2379-80.

12. Tenedios F, Erkan D. Cardiac involvement in the antiphospholipid syndrome. Lupus 2005;4:691-6.

13. Weening JJ, D'Agati VD, Schwartz MM, et al. The classification of glomerulonephritis in systemic lupus erythematosus revisited. J Am Soc Nephrol 2004;15:241-50.

14. Ponticelli C, Moroni G. Renal transplantation in lupus nephritis. Lupus 2005;14:95-8.

15. Kandala NB, Connock M, Grove A. Belimumab: a technnological advance for systemic lupus erythematosus patients? report of a systematic review and metaanalysis. BMJ Open 2013;3:pii:e002852. 